\title{
Duration of Contact Precautions for Acute-Care Settings
}

\author{
David B. Banach, MD, MPH; ${ }^{1, a}$ Gonzalo Bearman, MD, MPH; ${ }^{2, a}$ Marsha Barnden, RNC, MSN, CIC; ${ }^{3}$ \\ Jennifer A. Hanrahan, DO, MSc; ${ }^{4}$ Surbhi Leekha, MBBS, MPH; ${ }^{5}$ Daniel J. Morgan, MD, MS; ${ }^{5}$ Rekha Murthy, MD; ${ }^{6}$ \\ L. Silvia Munoz-Price, MD, PhD; $;^{7}$ Kaede V. Sullivan, MD, MSc; ${ }^{8}$ Kyle J. Popovich, MD, MS; ${ }^{9}$ Timothy L. Wiemken, PhD $^{10}$
}

\section{P URPOSE}

This expert guidance document (EG) provides recommendations regarding discontinuation of contact precautions $(\mathrm{CP})$ at the individual patient level in acute-care hospitals employing $\mathrm{CP}$ for 1 or more of the following organisms: methicillin-resistant Staphylococcus aureus (MRSA), vancomycin-resistant enterococci (VRE), Clostridium difficile, and multidrug-resistant Enterobacteriaceae (MDR-E), including carbapenem-resistant Enterobacteriaceae (CRE) and extended-spectrum $\beta$-lactamase (ESBL)-producing organisms. This document also provides a review of the role of molecular testing in guiding decisions pertaining to duration of $\mathrm{CP}$ for patients with these organisms. The guidance does not address decisions regarding the initiation of CP for any specific organism.

Previously published guidelines describe components of $\mathrm{CP}$ and identify situations in which $\mathrm{CP}$ should be used; currently, however, few publications address the issue of how long CP should be maintained. At the time of publication, decisions related to implementation of $\mathrm{CP}$ for select, endemic organisms are made by individual facilities based on factors such as institutional epidemiology, resources, organizational priorities, and previously published guidance, and these vary widely. The SHEA Guidelines Committee (GLC) selected this topic to address when $\mathrm{CP}$ should be discontinued for individual patients in acute-care settings that employ $\mathrm{CP}$ for the aforementioned organisms.

Although the organisms addressed are frequently encountered in other settings (eg, nursing homes, long-term acute-care facilities, rehabilitation centers, outpatient medical care settings), additional considerations may affect the application of these recommendations outside the acute-care hospital environment.

\section{A UTHORS}

The authors consist of current and past members of the SHEA Guidelines Committee (GLC), who serve as volunteers. All authors are involved at their respective institutions in the development of policies pertaining to $\mathrm{CP}$, either directly or in an advisory role.

\section{INTENDED USE}

Special-topic EG documents are developed to address areas of relatively narrow scope that lack the level of evidence required for a formal guideline but are important for the provision of safe and effective healthcare. As such, systematic grading of evidence level is not provided for individual recommendations. Each EG is based on a synthesis of limited evidence, theoretical rationale, current practices, practical considerations, the opinion of the writing group, and consideration of potential harms where applicable. Within the EG, a summary list of recommendations is provided, along with their respective rationales. We also conducted a survey of the SHEA Research Network (SRN).

No EG can anticipate all situations and this EG is not meant to be a substitute for individual judgment by qualified professionals.

\section{METHODS}

\section{Expert Guidance Development}

This EG follows the process outlined in the Handbook for SHEA-Sponsored Guidelines and Expert Guidance Documents..$^{10}$ The topic of duration of $\mathrm{CP}$ was among those proposed and ranked highest by the GLC. A manuscript proposal was approved by the SHEA Publications Committee and the SHEA Board of Trustees. We developed PICO-style (ie, population, intervention, control, and outcomes) questions based on agreed-upon themes. These questions were used to define the scope of the EG and the development of search terms, which were voted on until unanimous approval was achieved. We identified the period during which articles would be collected as January 1, 1990, to April 1, 2016. Only English-language articles were included. The lists of articles generated by the searches were reviewed for inclusion by a primary reviewer and a secondary reviewer. The EG was also informed by a survey of the SHEA Research Network (SRN).

Affiliations: 1. University of Connecticut, Farmington, Connecticut; 2. Virginia Commonwealth University, Richmond, Virginia; 3. Adventist Health, Roseville, California; 4. MetroHealth Medical Center, Cleveland, Ohio; 5. University of Maryland, Baltimore, Maryland; 6. Cedars-Sinai Medical Center, Los Angeles, California; 7. Medical College of Wisconsin, Milwaukee, Wisconsin; 8. Temple University School of Medicine, Philadelphia, Pennsylvania; 9. Rush University Medical Center, Chicago, Illinois; 10. University of Louisville School of Public Health and Information Sciences, Louisville, Kentucky.

${ }^{a}$ Co-chairs.

Received October 10, 2017; accepted October 26, 2017; electronically published January 11, 2018

(c) 2018 by The Society for Healthcare Epidemiology of America. All rights reserved. 0899-823X/2018/3902-0001. DOI: 10.1017/ice.2017.245 
Expert guidance development includes a formalized process for reaching expert consensus. Recommendations are listed with rationale statements. The consensus around each recommendation was determined via an anonymous rating and comment period.

This EG was reviewed and approved by the SHEA Guidelines Committee, the SHEA Publications Committee, and the SHEA Board of Trustees. This EG was endorsed by the Association for Professionals in Infection Control and Epidemiology (APIC), the Society of Hospital Medicine (SHM), and the Association of Medical Microbiology and Infectious Disease Canada (AMMI Canada).

\section{Multidrug-Resistant Enterobacteriaceae (MDR-E)}

For purposes of this EG, we considered MDR-E to be Enterobacteriaceae described as ESBL-producing (ESBL-E), carbapenem-resistant (CRE), or MDR-E defined by resistance to multiple classes of antibiotics. This EG does not address additional epidemiologically significant multidrug-resistant Gram-negative organisms such as Pseudomonas aeruginosa or members of the Acinetobacter baumannii complex.

\section{Microbiology and Testing}

For this EG, we reviewed FDA-cleared microbiology tests. We recognize that other tests, including laboratory developed tests, are in use. These are not included. This document focused on MRSA, VRE, and MDR-E. Molecular testing for C. difficile is not addressed.

\section{Colonization}

Colonization was defined as the isolation of the organism from a nonsterile site in the absence of symptoms of infection.

\section{GUIDANCE STATEMENT}

This document does not provide recommendations regarding indications for the use of CP. It is intended for acute-care hospitals that already use $\mathrm{CP}$, and it addresses when and under what circumstances CP may be discontinued.

\section{Methicillin-Resistant Staphylococcus aureus (MRSA)}

\section{Recommendations.}

1. If a hospital uses $\mathrm{CP}$ for patients previously colonized or infected with MRSA, we recommend establishing a policy for the discontinuation of CP for MRSA.

2. For patients not on antimicrobial therapy with activity against MRSA, we recommend negative screening cultures to guide decisions about discontinuation of CP. The optimal number of negative cultures needed is unclear, though 1-3 negative cultures are often used. The anterior nares are a common site of culture sampling, though the literature is unclear regarding the optimal site and the role of extra-nasal sampling.
3. For high-risk patients, such as those with chronic wounds or patients from long-term care facilities, we recommend extending CP from the last MRSA-positive culture, prior to assessing for $\mathrm{CP}$ discontinuation.

4. Outside an outbreak setting, if a facility's endemic rates of MRSA infection are low, the hospital may consider the alternative approach of using $\mathrm{CP}$ for patients with active MRSA infection for the duration of the index admission and discontinuing $\mathrm{CP}$ on hospital discharge. In adopting this approach, a hospital should monitor facility MRSA infection rates, maximize and consider monitoring use of standard precautions, and minimize patient cohorting to avoid intrafacility transmission. If the hospital's MRSA infection rates increase, the hospital should transition to a screeningculture-based approach for discontinuation of CP.

Rationale. Wide variation exists in hospital practices for discontinuation of CP for MRSA, ${ }^{11}$ and a policy for discontinuation can provide guidance to practitioners and infection prevention staff. A policy for CP discontinuation should address (1) inclusion and exclusion criteria, (2) laboratory testing and surveillance strategies, and (3) policy implementation and oversight. The studies utilized for this manuscript used various time periods during which an individual with a history of MRSA could be eligible for testing for discontinuation of CP. The SRN survey found that most institutions used 1-3 negative MRSA surveillance cultures to determine whether $\mathrm{CP}$ could be discontinued, though some providers report that their hospital utilizes $\mathrm{CP}$ indefinitely in patients with a history of MRSA. Published evidence indicates that most patients will remain negative for MRSA colonization if they have 3 consecutive negative weekly surveillance cultures, ${ }^{12}$ though the optimal number of and interval between cultures remains unclear in the literature. Patients with chronic wounds and those from long-term care facilities may be at higher risk for persistent MRSA colonization and recolonization ${ }^{13}$; thus, for these patients, hospitals may extend CP. The optimal duration of extension is unknown, though a minimum of 6 months was used commonly by institutions in the SRN survey.

\section{Vancomycin-Resistant Enterococci (VRE)}

\section{Recommendations.}

1. If a hospital uses $\mathrm{CP}$ when caring for patients colonized or infected with VRE, we recommend establishing a policy for discontinuation of CP for VRE.

2. We recommend that following treatment of VRE infection, the hospital use negative stool or rectal swab cultures to guide decisions about the discontinuation of $\mathrm{CP}$. The optimal number of negative cultures needed is unclear, though 1-3 negative cultures, each at least 1 week apart if multiple cultures are obtained, are often used.

3. Hospitals should consider extending CP prior to assessing for CP discontinuation in VRE infected patients who are (1) highly immunosuppressed, (2) receiving broad 
spectrum systemic antimicrobial therapy without VRE activity, (3) receiving care in protected environments (eg, burn units, bone marrow transplant units, or settings with neutropenic patients), or (4) receiving care at institutions with high rates of VRE infection.

4. Outside an outbreak setting and if facility endemic rates of VRE infection are low, the hospital may consider the alternative approach of using CP for patients with active VRE infection for the duration of the index admission and discontinuation of $\mathrm{CP}$ on hospital discharge. In adopting this approach, hospitals should monitor VRE infection rates, maximize and consider monitoring use of standard precautions, and minimize patient cohorting to avoid intrafacility transmission. If institutional VRE infection rates increase, the hospital should transition to a screeningculture-based approach for discontinuation of CP.

Rationale. Wide variation exists in hospital practices regarding the duration of CP for patients with VRE infection or colonization, and a policy on discontinuation of can guide providers and infection control staff. A policy for CP discontinuation should address (1) inclusion and exclusion criteria, (2) laboratory testing and surveillance strategies, and (3) policy implementation and oversight. In the SRN survey, most institutions use 1-3 negative surveillance cultures to identify patients for which CP can be discontinued. Data on duration of VRE colonization indicate that colonization may be prolonged, with high rates of relapse after multiple consecutive negative surveillance cultures. ${ }^{14-16}$ Factors associated with prolonged VRE carriage include immunocompromising conditions and concomitant antibiotic exposure. ${ }^{16-19}$ Additionally, patients with diarrhea and uncontrolled respiratory secretions and draining wounds may pose the highest risk for transmission in the healthcare environment. The sensitivity of surveillance cultures of stool or rectal swab samples for the detecting VRE colonization is not well established. Settings where VRE infection rates increase, particularly in outbreak settings, or settings where care is provided to patients who, if colonized, may be at high risk for invasive infection, may benefit from intensified infection prevention efforts to reduce VRE transmission. Most frequently, 3 consecutive negative cultures performed weekly have been studied, although hospitals may consider other strategies. ${ }^{14,20}$ Given the limitations of sequential culture strategies in documenting VRE eradication, prolonging $\mathrm{CP}$ may be an effective enhanced measure in preventing VRE transmission in high-risk settings, though evidence does not exist to identify the optimal period of prolongation.

\section{Multidrug-Resistant Enterobacteriaceae (MDR-E)}

\section{Recommendations.}

1. If a hospital uses $\mathrm{CP}$ for patients infected or colonized with MDR-E (ESBL-E and/or CRE), we recommend establishing a policy for discontinuation of CP for MDR-E that includes the following:

a. Maintaining CP for ESBL-E and CRE for the duration of the index hospital stay when infection or colonization with these bacteria is first detected.

b. Considering discontinuation of $\mathrm{CP}$ on a case-by-case basis, taking into account the following criteria: (1) at least 6 months have elapsed since the last positive culture; (2) presence of a clinical infection and ongoing antibiotic use, where discontinuation of $\mathrm{CP}$ should be discouraged in the setting of suspected or known infection with ESBL-E or CRE, and concurrent broadspectrum antibiotic use that may select for these organisms; and (3) procurement of an adequate number of screening samples, with at least 2 consecutive negative rectal swab samples obtained at least 1 week apart to consider an individual negative for ESBL-E or CRE colonization.

2. We recommend that for extensively drug-resistant Enterobacteriaceae, such as carbapenemase-producing CRE, or Enterobacteriaceae with very limited treatment options (susceptible to $\leq 2$ antibiotic classes used to treat that organism), hospitals should maintain CP indefinitely.

Rationale. Variation exists among hospital practices regarding duration of CP for patients with ESBL-E and CRE infection or colonization, with most respondents in the survey reporting that their hospital utilizes CP indefinitely in patients with a history of ESBL or CRE. If an institution elects to consider discontinuation of CP for MDR-E, we recommend a policy to guide practitioners and infection prevention staff that includes (1) inclusion and exclusion criteria, (2) laboratory testing and surveillance strategies, and (3) policy implementation and oversight. Nearly all studies described prolonged and persistent colonization with these organisms. Various risk factors are associated with persistent carriage with ESBL-E and CRE and include positivity on clinical (vs screening) cultures and exposure and re-exposure to healthcare facilities. ${ }^{21,22}$ Studies also have described variability in detection of these bacteria from the gastrointestinal (GI) tract of the same individual over time (ie, positive culture followed by negative culture then reverting to positive). ${ }^{21,23}$ Finally, certain extensively drug-resistant Enterobacteriaceae, particularly carbapenemase-producing bacteria, have no or limited treatment options, which makes the impact of even a single transmission event significant and provides the basis for a more conservative approach to duration of $\mathrm{CP}$.

\section{Clostridium difficile}

\section{Recommendations.}

1. We recommend that patients with $C$. difficile infection (CDI) receive care with $\mathrm{CP}$ for at least 48 hours after resolution of diarrhea. 
2. Hospitals should consider extending $\mathrm{CP}$ through the duration of hospitalization if elevated rates of CDI are present despite appropriate infection prevention and control measures.

3. At this time, insufficient evidence exists to make a formal recommendation as to whether patients with CDI should be placed on CP if they are readmitted to the hospital.

Rationale. Currently, the Centers for Disease Control and Prevention (CDC) and the SHEA/IDSA Compendium of Strategies to Prevent Clostridium difficile in Acute-Care Hospitals recommend the discontinuation of CP 48 hours after the resolution of diarrhea among patients diagnosed with C. difficile colitis. ${ }^{24}$ Patients that are $C$. difficile carriers shed the organism in their stools for weeks after cessation of diarrhea. ${ }^{25,26}$ The shedding of $C$. difficile spores after resolution of diarrhea may contribute to the spread of this organism. Recent data suggest that isolation of asymptomatic carriers reduced the incidence of $C$. difficile in the hospital setting. ${ }^{27}$ Based on these findings, we recommend extending the duration of $\mathrm{CP}$ for the duration of hospitalization in settings in which control of C. difficile is not optimal despite the institution of the standard practices. At this time, evidence does not exist supporting repeat laboratory testing for $C$. difficile to guide decisions regarding discontinuation of CP for patients with CDI.

\section{Microbiological Screening and Molecular Testing}

Recommendation. At this time, insufficient evidence exists to make a formal recommendation supporting the use of molecular testing for the purpose of discontinuation of CP for MDROs.

Rationale. While we assume that polymerase chain reaction (PCR) tests perform with superior sensitivity compared to culture, due to lack of high-quality studies at this time, we cannot definitively ascertain the impact of molecular methods on informing the duration of colonization and guiding decisions about CP.

\section{B A C K GROUN D}

\section{SHEA Research Network Survey}

We surveyed SHEA Research Network (SRN) institutions to ascertain policies and practices for guiding duration and discontinuation of CP for MRSA, VRE, MDR-E, and C. difficile, as well as screening and testing methods (Table 1). On June 22, June 28 , and July 6,2016 , SHEA sent the survey to members of the SRN, which includes 134 individual institutions, $26 \%$ of which are outside the United States and Canada. Among them, 4 institutions opted out because they were not eligible to participate, and 7 indicated that they were unavailable to respond during the time the survey was open. The survey response rate was $70.7 \%$ ( 87 of 123 ).

Of the responding institutions, $60.5 \%$ were academic medical centers, $18.4 \%$ were community teaching hospitals with academic affiliations, and $7.9 \%$ were community hospitals with no academic affiliations. The responding institutions varied in bed size and number of intensive care units (ICUs): 51-300 beds (29.7\%), 301-600 beds (31.4\%), 601-900 beds (16.3\%), > 900 beds $(23.3 \%)$, and $1-3$ ICUs (41.9\%), 4-6 ICUs (30.2\%), 7-9 ICUs (12.8\%), $\geq 10$ ICUs (14\%). Overall, $94 \%$ of institutions had pediatric beds. On average, institutions reported having 1.4 hospital epidemiologists and 4.8 infection preventionists.

Individual respondents reported the following primary roles: healthcare epidemiologist $(75.3 \%)$, infection committee chair (54.6\%), and infection preventionist (26\%). These individuals indicated that they are involved in patient care $(40.3 \%)$, teaching $(40.3 \%)$, clinical research $(31.2 \%)$, and administration $(18.9 \%)$.

More than half of the respondents identified $C$. difficile (56\%) as the most problematic pathogen at their institution. Another 27\% reported that multidrug-resistant Gram-negative rods (MDR-GNR), such as ESBL and CRE, were their highest concern. MRSA and VRE followed with $14 \%$ and $2 \%$, respectively. Moreover, $38 \%$ of respondents reported a healthcare-associated cluster or outbreak related to C. difficile infection in calendar year 2015, followed by MDR-GNR (24\%), MRSA (17\%), and VRE (8\%).

Most respondents reported using $\mathrm{CP}$ in patients colonized with MDRO: CRE (99\%), MRSA (89\%), VRE (90\%), and other MDR-GNR (79\%). All respondents reported using CP for at least 1 indication. In addition, $75 \%$ of respondents reported that their institution had a policy for discontinuing $\mathrm{CP}$ in patients colonized with MRSA; $61 \%$ for VRE; $32 \%$ for CRE; and $40 \%$ for other MDR-GNR. Furthermore, $25 \%$ of respondents reported using molecular methods of testing for detection of MRSA; $9 \%$ for VRE; $8 \%$ for CRE; and 5\% for other MDR-GNR. The remainder reported the use of culture methods for screening.

Most respondents reported the use of screening tests for discontinuation of CP for MRSA (66\%) and VRE (55\%), but tests were less commonly used for discontinuing $\mathrm{CP}$ in patients with CRE and other MDR-GNR (29\% and 26\%, respectively). With regard to anatomic screening sites, the nares was the most commonly screened site for MRSA, followed by the rectum for VRE and CRE. In cases in which patients were infected with an MDRO, the original site of infection was screened frequently ( $45 \%$ for MRSA, $28 \%$ for VRE, $23 \%$ for CRE, and $21 \%$ for other MDR-GNR). Respondents reported using screening for discontinuing $\mathrm{CP}$ more frequently with MRSA and VRE ( $83 \%$ and $80 \%$, respectively) than with CRE and other MDR-GNR ( $73 \%$ and 63\%, respectively). Most commonly, 3 negative screens were required for discontinuing CP for MRSA (40\%), VRE (46\%), and CRE (14\%). A large proportion of respondents indicated that $>5$ days need to elapse between screening specimen collections ( $30 \%$ for MRSA, $42 \%$ for VRE, $17 \%$ for CRE, and $12.7 \%$ for MDR-GNR).

The use of decolonization was reported by nearly a quarter of respondents for the purpose of discontinuing CP in MRSA (24\%). 
T A B LE 1. Survey of Institutions in the SHEA Research Network (SRN)

\begin{tabular}{lcr}
\hline & The Most Problematic Pathogens at the Institution Based on the Infection Control Risk Assessment & $\%$ \\
\hline & No. & 14.3 \\
MRSA & 12 & 56.0 \\
CDI & 47 & 2.4 \\
VRE & 2 & 27.4 \\
CRE/ESBL & 23 & 96.6 \\
Total & 84 & 3.4 \\
No response & 3 & \\
\hline
\end{tabular}

Healthcare-Associated Cluster or Outbreak during Jan 1-Dec 31, 2015, Requiring Infection Prevention Resources (eg, investigation, control, etc.) Caused by 1 of the Following Pathogens

\begin{tabular}{lrr}
\hline & No. & $\%$ \\
\hline MRSA & 15 & 17.2 \\
CDI & 33 & 37.9 \\
VRE & 7 & 8.0 \\
CRE/ESBL & 21 & 24.1 \\
No clusters/outbreaks & 31 & 35.6 \\
\hline
\end{tabular}

CP Used for Patients Documented to Have Colonization or Infection with 1 of the Following Organisms

\begin{tabular}{lcc}
\hline & No. & $\%$ \\
\hline MRSA & 77 & 88.5 \\
VRE & 78 & 89.7 \\
CRE/ESBL & 86 & 98.9 \\
Non-CRE MDR-GNR & 69 & 79.3 \\
No CP for any MDRO & 0 & 0.0 \\
\hline
\end{tabular}

No CP for any MDRO

Institution Has a Policy That Allows for Discontinuation of CP for These Organisms

\begin{tabular}{lcc}
\hline & No. & $\%$ \\
\hline MRSA & 65 & 74.7 \\
VRE & 53 & 60.9 \\
CRE/ESBL & 28 & 32.2 \\
Non-CRE MDR-GNR & 35 & 40.2 \\
\hline
\end{tabular}

Institution Screens or Tests for Discontinuing CP

\begin{tabular}{lcc}
\hline & No. & $\%$ \\
\hline MRSA & 57 & 65.5 \\
VRE & 48 & 55.2 \\
CRE/ESBL & 25 & 28.7 \\
Non-CRE MDR-GNR & 34 & 39.1 \\
\hline
\end{tabular}

Institution Uses a Decolonization Regimen to Discontinue CP

\begin{tabular}{lcc}
\hline & No. & $\%$ \\
\hline MRSA & 18 & 20.7 \\
VRE & 3 & 3.4 \\
CRE/ESBL & 2 & 2.3 \\
Non-CRE MDR-GNR & 2 & 2.3 \\
\hline
\end{tabular}

Institution Uses Time Since Last Positive Culture to Determine Whether to Discontinue CP

\begin{tabular}{lcc}
\hline & No. & $\%$ \\
\hline MRSA & 23 & 26.4 \\
VRE & 19 & 21.8 \\
CRE/ESBL & 16 & 18.4 \\
Non-CRE MDR-GNR & 19 & 21.8 \\
\hline
\end{tabular}


Sites Used for Screening to Discontinue CP (check all that apply)

\begin{tabular}{|c|c|c|c|c|c|c|c|c|}
\hline & \multicolumn{2}{|c|}{ MRSA } & \multicolumn{2}{|c|}{ VRE } & \multicolumn{2}{|c|}{ CRE } & \multicolumn{2}{|c|}{ ESBL } \\
\hline Original site & 40 & 46.0 & 24 & 27.6 & 20 & 23.0 & 18 & 20.7 \\
\hline Perirectal & 11 & 12.6 & 48 & 55.2 & 18 & 20.7 & 11 & 12.6 \\
\hline Skin & 23 & 26.4 & 6 & 6.9 & 3 & 3.4 & 4 & 4.6 \\
\hline
\end{tabular}

No. of Negative Screening Tests Required to Discontinue CP

\begin{tabular}{|c|c|c|c|c|c|c|c|c|c|c|c|}
\hline & \multicolumn{2}{|c|}{1} & \multicolumn{2}{|c|}{2} & \multicolumn{2}{|c|}{3} & \multicolumn{2}{|c|}{$>3$} & \multicolumn{2}{|c|}{$\begin{array}{l}\text { Do Not Screen for } \\
\text { Discon-tinuation }\end{array}$} & \multirow{2}{*}{$\frac{\text { Total }}{\text { No. }}$} \\
\hline & No. & $\%$ & No. & $\%$ & No. & $\%$ & No. & $\%$ & No. & $\%$ & \\
\hline MRSA & 13 & 18.1 & 22 & 30.6 & 28 & 38.9 & 1 & 1.4 & 8 & 11.1 & 72 \\
\hline VRE & 5 & 7.1 & 13 & 18.6 & 32 & 45.7 & 3 & 4.3 & 17 & 24.3 & 70 \\
\hline CRE & 6 & 9.5 & 8 & 12.7 & 9 & 14.3 & 3 & 4.8 & 37 & 58.7 & 63 \\
\hline
\end{tabular}

Minimum Time Interval Between Specimen Collections When Testing to Discontinue CP

\begin{tabular}{|c|c|c|c|c|c|c|c|}
\hline & \multicolumn{2}{|c|}{$\leq 5$ Days } & \multicolumn{2}{|c|}{$>5$ Days } & \multicolumn{2}{|c|}{ Variable } & \multirow{2}{*}{$\frac{\text { Total }}{\text { No. }}$} \\
\hline & No. & $\%$ & No. & $\%$ & No. & $\%$ & \\
\hline VRE & 23 & 33.3 & 29 & 42.0 & 17 & 24.6 & 69 \\
\hline CRE & 37 & 56.9 & 11 & 16.9 & 17 & 26.2 & 65 \\
\hline ESBL & 13 & 23.6 & 7 & 12.7 & 35 & 63.6 & 55 \\
\hline
\end{tabular}

Patients Required to be off Antibiotics with Activity Against the Organism of Interest Prior to Repeat Screening for Discontinuation of CP

\begin{tabular}{lcc}
\hline & No. & $\%$ \\
\hline Yes & 49 & 56.3 \\
No & 25 & 28.7 \\
No response & 13 & 14.9 \\
\hline
\end{tabular}

Time Interval Used From Discontinuation of Antimicrobial Therapy Until a Specimen is Obtained for the Purposes of Removal of CP

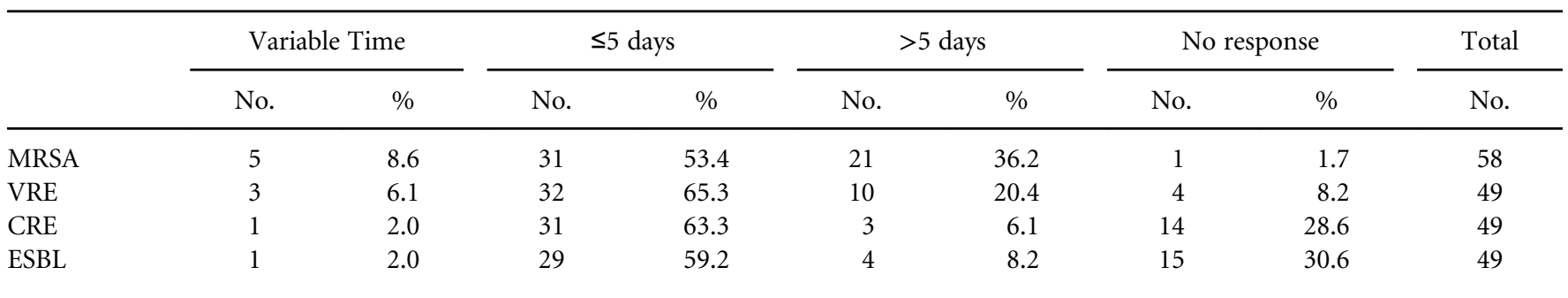

CP Discontinued Based on the Length of Time Since the Last Positive Laboratory Test

\begin{tabular}{lll}
\hline & No. & $\%$ \\
\hline Yes & 28 & 32.2 \\
No & 49 & 56.3 \\
No response & 10 & 11.5 \\
\hline
\end{tabular}


Timeframe Used Since Last Positive Laboratory Test to Discontinue CP

\begin{tabular}{|c|c|c|c|c|c|c|c|c|c|c|c|c|c|}
\hline & \multicolumn{2}{|c|}{ Current Hospital-ization } & \multicolumn{2}{|c|}{1 Month } & \multicolumn{2}{|c|}{6 Months } & \multicolumn{2}{|c|}{1 Year } & \multicolumn{2}{|c|}{$>1$ Year } & \multicolumn{2}{|c|}{ No Response } & $\frac{\text { Total }}{\text { No. }}$ \\
\hline VRE & 4 & 20.0 & 1 & 5.0 & 5 & 25.0 & 7 & 35.0 & 3 & 15.0 & 8 & 28.6 & 28 \\
\hline CRE & 1 & 6.3 & 0 & 0.0 & 4 & 25.0 & 5 & 31.3 & 6 & 37.5 & 12 & 42.9 & 28 \\
\hline ESBL & 4 & 19.0 & 2 & 9.5 & 6 & 28.6 & 4 & 19.0 & 5 & 23.8 & 7 & 25.0 & 28 \\
\hline
\end{tabular}

Testing Method Used to Confirm Clearance (check all that apply)

\begin{tabular}{|c|c|c|c|c|c|}
\hline & \multicolumn{2}{|c|}{ Culture } & \multicolumn{2}{|c|}{ PCR } & \multirow{2}{*}{$\frac{\text { Total }}{\text { No. }}$} \\
\hline & No. & $\%$ & No. & $\%$ & \\
\hline VRE & 59 & 91 & 6 & 9 & 65 \\
\hline CRE & 24 & 92 & 2 & 8 & 26 \\
\hline ESBL & 21 & 95 & 1 & 5 & 22 \\
\hline
\end{tabular}

When CP for C. difficile is Stopped

\begin{tabular}{lrr}
\hline & No. & $\%$ \\
\hline Upon discharge & 34 & 40.5 \\
When diarrhea stops & 4 & 4.8 \\
At least 24 hours after diarrhea stops & 38 & 45.2 \\
1 C. difficile testing negative & 1 & 1.2 \\
$>1$ C. difficile test negative & 3 & 3.6 \\
Never & 2 & 2.4 \\
CP not used for CDI & 2 & 2.4 \\
No response & 3 & NA \\
\hline
\end{tabular}

Action When a Patient is Readmitted After a Diagnosis of C. difficile Within the Prior 6 Months

\begin{tabular}{lrr}
\hline & No. & $\%$ \\
\hline CP started regardless of symptoms & 8 & 9.3 \\
CP started if diarrhea & 41 & 47.1 \\
Manage as any other admission & 37 & 42.5 \\
No response & 1 & 1.1 \\
\hline
\end{tabular}

How Institution Uses PCR and/or EIA for C. difficile Testing (check all that apply)

\begin{tabular}{|c|c|c|c|c|}
\hline & \multicolumn{2}{|c|}{ Clinical } & \multicolumn{2}{|c|}{$\mathrm{CP}$} \\
\hline & No. & $\%$ & No. & $\%$ \\
\hline EIA & 37 & 42.5 & 4 & 4.6 \\
\hline
\end{tabular}

NOTE. CP, contact precautions; MRSA, methicillin-resistant Staphylococcus aureus; VRE, vancomycin-resistant enterococci (VRE); CDI, Clostridium difficile infection; MDR-GNR, multidrug-resistant Gram-negative rods; CRE, carbapenem-resistant Enterobacteriaceae; ESBL, extended-spectrum $\beta$-lactamase; EIA, enzyme immunoassay; PCR, polymerase chain reaction.

A small number of institutions reported its use for eradication of VRE (3\%), CRE (2\%), and other MDR-GNR (2\%).

Respondents reported that $\mathrm{CP}$ for $C$. difficile infection (CDI) is discontinued most frequently upon discharge $(40 \%)$ or $\geq 24$ hours after the cessation of diarrhea related to CDI (45\%). Patients who are admitted with a history (within the last
6 months) of CDI are most frequently put on CP if they develop diarrhea $(47 \%)$ or are managed as if they do not have a history of CDI (42\%). In addition, $85 \%$ of respondents reported use of $C$. difficile toxin PCR for diagnosis of CDI, and $42 \%$ reported the use of enzyme immunoassay (EIA) for this purpose, suggesting that some institutions may be using both. 
Less than 5\% of PCR and EIA users reported their use for discontinuing $\mathrm{CP}$.

\section{METHICILLIN-RESISTANT STAPHYLOCOCCUS AUREUS (MRSA)}

\section{Current Recommendations on the Discontinuation of CP}

Several states have laws supporting screening programs to identify patients with MRSA colonization, ${ }^{28}$ particularly patients viewed to be high-risk for MRSA colonization (eg, patients admitted to the intensive care unit). ${ }^{29}$ Hospitals determine where and when active surveillance should be performed and which high-risk populations and body sites are tested for colonization. ${ }^{30}$ In addition, some hospitals perform surveillance cultures during hospitalization in predefined units to identify cases of MRSA acquisition. ${ }^{30}$ Because colonization with MRSA can be persistent, ${ }^{31}$ especially in high-risk populations, some previously published guidance documents recommend automatically placing patients on $\mathrm{CP}$ during hospitalization if they have a history of MRSA colonization or infection during prior hospital admissions, ${ }^{5,30}$ thereby increasing the population in healthcare facilities who are on CP for MRSA. ${ }^{5}$

There are no national guidelines pertaining to discontinuation of MRSA CP. The optimal duration of CP is unknown for patients previously colonized or infected with MRSA. Institutions often develop their own criteria for the discontinuation of $\mathrm{CP}^{9,11,32}$ Siegel et $\mathrm{al}^{33}$ suggest that if a patient has not been on antibiotics for several weeks and has at least 3 negative surveillance cultures for MRSA over the course of 1-2 weeks, this may be sufficient for discontinuing CP.

Variation exists among hospital policies for the discontinuation of CP for MRSA. Shenoy et $\mathrm{al}^{11}$ conducted a survey of infection preventionists on the discontinuation of $\mathrm{CP}$ and found that $73 \%$ of respondents stated that their institutions had a policy for discontinuing $\mathrm{CP}$ for patients with a history of MRSA. However, the study found significant variation in the criteria used, and most institutions did not actively screen patients for the purpose of $\mathrm{CP}$ discontinuation. A survey of 55 hospitals administered by Hospital Corporation of America (HCA, Inc.), a large healthcare system in the United States, found that $68 \%$ of ICUs had a policy for discontinuing CP for MRSA. ${ }^{34}$ Additionally, $18 \%$ of the respondents to a survey administered to physicians in the Emerging Infections Network (EIN) reported that the duration of isolation for MRSA at their hospital was indefinite once a patient was positive. ${ }^{9}$

Shenoy et $\mathrm{al}^{36}$ also conducted a randomized control trial of individuals with a history of MRSA colonization or infection at least 90 days prior to enrollment in the study and compared standard hospital practice of clinician-initiated screening using 3 sequential cultures (ie, "passive screening") to study initiated screening for discontinuation of CP (ie, "active screening"). In the active screening arm, research staff obtained 3 sets of surveillance nasal cultures performed at least 24 hours apart, processed by both culture and PCR. Patients in both arms with 3 negative cultures who had not received antibiotics with activity against MRSA were eligible to have CP discontinued. The investigators found that in the passive screening arm, $31 \%$ of subjects had screening initiated and only $9.6 \%$ of subjects completed the series of swabs. In contrast, all subjects in the active screening arm had screenings initiated, and $74 \%$ had 3 swabs obtained to complete the series. ${ }^{36}$ The negative predictive value of the first nasal swab processed by PCR was $97 \%$ compared to 3 sequential cultures. Shenoy et $\mathrm{al}^{37}$ also evaluated the use of a single PCR-based screening in an emergency department for patients with a history of MRSA prior to 90 days from that visit and who were not receiving antibiotics with activity against MRSA in the prior 48 hours. Of the patients eligible for the study, $65 \%$ were PCR negative and CP were discontinued. However, $69 \%$ of patients were not properly assessed for enrollment, demonstrating the significant challenges in implementing active screening programs for CP discontinuation, particularly in busy emergency departments. ${ }^{38}$

Other studies evaluating screening for discontinuation of $\mathrm{CP}$ used longer time intervals since the last MRSA culture for study eligibility. In a study by Vikram et $\mathrm{al}^{39}$ individuals with no positive MRSA cultures in 6 months prior to hospital admission were screened for MRSA at multiple body sites (nares; wound if present; and if no wound, axillae and perineum) 3 consecutive times. The authors observed that only $21 \%$ of patients had 3 sets of surveillance cultures that were negative and were removed from CP. Goldsack et a ${ }^{40}$ collected 2 sets of nares cultures from patients readmitted to the hospital with a history of MRSA at least 1 year prior to the admission and without receipt of antibiotics in the prior 72 hours to assess continued MRSA colonization. They found that $80 \%$ of patients studied were no longer colonized with MRSA; these patients were subsequently removed from contact isolation. ${ }^{40}$

Automated electronic health record systems with microbiologic data and electronic alerts may improve compliance with hospital policies for CP. In one system, discontinuation criteria are displayed to physicians when they attempt to discontinue $\mathrm{CP}$, and only an infection preventionist can remove the visual alert for MRSA in the electronic health record when criteria are met per hospital protocol. ${ }^{41}$ Carson et $\mathrm{al}^{42}$ evaluated outpatients who were not on long-term antibiotics and who had a MRSA infection at least 3 months prior to enrollment and therefore were "MRSA flagged" in the EMR. Eligible subjects had surveillance cultures obtained from the nares, axilla, inguinal area, and wound if present; if all cultures were negative, patients returned at least 7 days later for testing at these body sites a second time. They observed that $72 \%$ of outpatients in the study were no longer carriers of MRSA and were able to have the alert removed from their medical record. ${ }^{42}$

\section{Duration of Colonization}

Staphylococcus aureus colonization may be persistent (always present), intermittent (sometimes present and sometimes 
absent), or rarely occurring. ${ }^{31}$ Studies examining individuals following hospital discharge have suggested that over time, many individuals will clear MRSA colonization, although a proportion will remain long-term carriers. ${ }^{13,43,44}$ Long-term carriers of MRSA retain an increased risk of serious infection and even death due to MRSA compared to noncarriers. ${ }^{45}$ The median time to clearance of MRSA colonization is 7-9 months ${ }^{13,46,47}$; however, some individuals can have more prolonged carriage. ${ }^{43}$ In an evaluation of children in the community over a 1-year period, $18 \%$ of children who were colonized with MRSA in the nares at enrollment remained colonized 1 year later. ${ }^{48}$ In a retrospective assessment of known MRSA carriers who were readmitted to a large tertiary-care hospital, Sanford et $\mathrm{al}^{44}$ observed that a proportion of readmitted carriers remained colonized for $>3$ years and that all patients colonized for $>12$ months had at least 1 positive wound culture. Similarly, Scanvic et $\mathrm{al}^{13}$ examined patients who were previously identified as MRSA carriers who were readmitted to the hospital and reported that those with a break in the skin at readmission were more likely to have persistent carriage of MRSA. Other factors associated with prolonged carriage of MRSA include older age, household members with MRSA colonization, need for help with daily activities, indwelling devices, a recent ICU stay, less time since the last MRSA-positive culture, longer length of stay in a healthcare facility, or residence in a long-term care facility. ${ }^{42,44,47,49-58}$ In addition, colonization with MRSA at multiple body sites has been associated with prolonged carriage among patients readmitted to a university hospital. ${ }^{59}$

Some studies have documented shorter duration of colonization. Ghosh et $\mathrm{al}^{60}$ examined patients with prolonged hospital stays and used active surveillance to identify patients that lose MRSA carriage. They observed a clearance rate of $11 \%$ for MRSA with a median time to clearance of 23 days, although 4 of these 19 patients were later found to be recolonized with MRSA. Cluzet et $\mathrm{al}^{49}$ also documented a shorter duration of MRSA colonization of 21 days in individuals screened every 2 weeks following a skin infection due to MRSA. Notably, this population was younger, and most individuals received antibiotic therapy for skin infection, which could contribute to earlier clearance; $20 \%$ of individuals never cleared colonization during 6 months of follow-up. In a separate analysis, these investigators observed that $43.6 \%$ of individuals who had cleared MRSA colonization ultimately became recolonized with a median time to recurrence of 53 days. ${ }^{61}$ In a study of patients at neurologic long-term care facilities, patients not colonized with $S$. aureus at admission were followed with weekly nasal surveillance cultures for a median duration of 20 weeks. The median time to clearance of MRSA colonization (acquisition of colonization until 2 consecutive nasal swabs negative for that strain) was 3 weeks. ${ }^{62}$

Challenges exist to developing a standardized definition of clearance of MRSA. Due to the heterogeneity among study designs, it is difficult to draw overarching conclusions. Prior studies showed considerable variability with respect to follow-up time, frequency of screening cultures, and definitions of what constituted MRSA clearance. Some studies included potential confounding variables, such as receipt of concomitant antibiotics or the use of decolonization regimens. ${ }^{63}$ Because of variations in MRSA colonization duration, many hospitals wait at least 3 months prior to assessing an individual for clearance of MRSA colonization, ${ }^{36}$ with a large proportion of hospitals requiring 3 negative specimens to document clearance of MRSA. ${ }^{36}$ Published evidence indicates that most patients will remain negative for MRSA if they have 3 consecutive negative weekly surveillance cultures. ${ }^{12}$

\section{Sites of Colonization}

The anterior nares is the primary site of MRSA colonization, ${ }^{31}$ although various extra-nasal sites are also colonized (eg, throat, axilla, inguinal area, perirectal area, and chronic wounds). ${ }^{64}$ Most hospital surveillance programs screen for MRSA colonization solely in the anterior nares. However, this screening may result in unrecognized extra-nasal colonization. ${ }^{30}$ A review of 23 studies on testing for MRSA colonization estimated that extra-nasal screening increased MRSA detection by more than $33 \%$ compared to nares screening alone. ${ }^{64}$ It is unclear whether evaluation of extra-nasal MRSA colonization is beneficial for hospital surveillance programs or if extra-nasal colonization should be considered in a decision about discontinuation of $\mathrm{CP}$. Some hospitals use nares cultures alone to confirm clearance, whereas others use additional sites of cultures to guide protocols for discontinuing CP. ${ }^{11}$ Further research is warranted in the role of extra-nasal surveillance cultures for the purpose of discontinuing $\mathrm{CP}$.

\section{VANCOMYCIN-RESISTANT ENTEROCOCCUS (VRE)}

\section{Current Recommendations on the Discontinuation of CP}

Early guidance suggested that CP for VRE should be discontinued after 3 negative stool cultures obtained at least 1 week apart. ${ }^{65}$ Subsequent guidelines from CDC and HICPAC supported this practice in the absence of uncontrolled respiratory secretions, draining wounds, or the involvement of the patient in an institutional outbreak. ${ }^{1}$ Sequential testing revealed that after 3 sequential negative cultures, 35 of 37 patients (95\%) remained culture negative. ${ }^{20}$ One institution, which defined VRE clearance as 3 negative stool cultures more than 3 weeks apart, found in subsequent, prospective surveillance testing that VRE recolonization occurred in 5 of 21 patients (24\%) who had "cleared."14 The same authors identified 12 patients who had at least 2 VRE-positive cultures more than 1 year apart, supporting concern for prolonged colonization. ${ }^{14}$

Donskey et $\mathrm{al}^{15}$ demonstrated that individuals who had cleared VRE colonization with 3 negative stool cultures collected at least 1 week apart had a high recurrence rate $(62 \%)$ if they were exposed to antibiotics in the following year. Individuals with risk factors for VRE colonization have demonstrated recurrence, ${ }^{16}$ and recent studies suggest that a strategy 
of 3 weekly consecutive surveillance cultures to identify VRE clearance results in recurrence rates of $11 \% .{ }^{66}$

\section{Duration of Colonization}

Multiple studies evaluated the duration of VRE colonization and the predictive value of VRE-negative stool cultures in predicting VRE clearance. Studies on this subject have been largely retrospective and have been focused in hospital settings. A retrospective analysis of a large, multicenter study revealed that among 394 patients with VRE colonization, $76 \%$ of those admitted within 50 days of initial VRE detection remained positive for VRE, and among 126 patients admitted over 300 days from the last positive VRE culture, 20 (15.9\%) remained positive in screening surveillance cultures. ${ }^{67}$ In a single-center study, 35 of 210 VRE-colonized patients cleared colonization at a mean of 49 days. Using a logistic regression model the study predicted that $40 \%$ of colonized patients would clear colonization at 100 days. ${ }^{68}$ In another study, 22 of 105 VRE colonized patients remained colonized for more than 100 days. ${ }^{20}$ A recent systematic analysis pooled data on the subject from 13 studies, identifying a range of VRE clearance from 1 to 43 weeks. A logistic regression model estimated that $50 \%$ of subjects cleared colonization at 25 weeks after an initial VRE-positive stool test. ${ }^{63}$ Limitations associated with most of these studies include inconsistent reporting of concomitantly administered antibiotics, variation in testing modalities (culture vs molecular methods), limited duration of follow-up, and retrospective nature of the study designs.

Several factors may influence the duration of VRE colonization and the likelihood of VRE transmission in the hospital setting. Prolonged hospitalization and intensive care unit stay have been associated with prolonged VRE colonization. ${ }^{20}$ Immunosuppressed patients may remain colonized with VRE for longer periods of time $e^{17,18,69}$ and may have a high rate of recurrent VRE colonization after multiple negative VRE cultures. ${ }^{16}$ Antibiotic use, specifically vancomycin ${ }^{19,70}$ and fluoroquinolone ${ }^{17,19}$ use, have been associated with prolonged VRE colonization. Older patients and patients receiving fluoroquinolones were more likely to transmit VRE to roommates. ${ }^{71}$ Prior guidance suggests consideration of concurrent antibiotic use, particularly antibiotics with activity against VRE, when making decisions regarding discontinuation of $\mathrm{CP} .{ }^{1}$

\section{Sites of Colonization}

The gastrointestinal tract is the main site of VRE colonization, though VRE has been identified on the skin of patients colonized with VRE with diarrhea. ${ }^{72}$ Most studies investigating the duration of VRE colonization duration have used gastrointestinal tract samples, ${ }^{63}$ stool samples, ${ }^{14,19}$ and perirectal and perineal swabs, ${ }^{20,67,68,73}$ or both. ${ }^{12,60}$ Some studies have included surveillance cultures obtained from other sites including throat and urine samples. ${ }^{16}$ While limited, data comparing the sensitivity of rectal and perirectal cultures suggest that both are comparable. ${ }^{74}$
MULT IDRUG-RES IST A N T ENTEROBACTERIACEAE (MDR-E)

Current Recommendations Regarding the Discontinuation of $\mathrm{CP}$

The CDC HICPAC MDRO guidelines state that "In general, it seems reasonable to discontinue $\mathrm{CP}$ when 3 or more surveillance cultures for the target MDRO are repeatedly negative over the course of 1 or 2 weeks in a patient who has not received antimicrobial therapy for several weeks, especially in the absence of a draining wound, profuse respiratory secretions, or evidence implicating the specific patient in ongoing transmission of the MDRO within the facility." The CDC CRE Tool Kit 2015 update states that "Currently, there is not enough evidence to make a firm recommendation about when to discontinue use of $\mathrm{CP}$ for infected or colonized patients; however, CRE colonization can be prolonged ( $>6$ months). If surveillance cultures are used to decide if a patient remains colonized, $>1$ culture should be collected to improve sensitivity. Regardless of whether surveillance cultures are performed, the presence of risk factors for ongoing carriage or ongoing CRE exposure should be considered in the decision about discontinuing CP." ${ }^{75}$

In a survey among SHEA Research Network members, among 49 respondents, CP for patients infected with ESBL-E were reportedly maintained for the duration of active illness by $8.2 \%$ of respondents, for the duration of hospitalization by $26.5 \%$, until negative surveillance cultures were obtained by $32.7 \%$, and indefinitely by $34.7 \%{ }^{76}$ Overall, $55 \%$ of respondents reported isolating patients at readmission. For those respondents requiring negative cultures for "clearance" $(n=16)$, cultures were obtained after completion of antibiotics by $37.5 \%$ of respopndents, after hospital discharge by $25 \%$, and within 3 months by $12.5 \%$. Among 62 respondents regarding CRE, CP were reportedly maintained for the duration of active illness by $6.5 \%$ of respondents, for the duration of hospitalization by $12.9 \%$, until negative surveillance cultures by $29 \%$, and indefinitely by $43.5 \%$. Furthermore, $72 \%$ of respondents instituted isolation at readmission for CRE. Among respondents reporting that they require surveillance cultures for CRE clearance $(n=18)$, cultures could be obtained after completion of antibiotics by $44.4 \%$ respondents, after hospital discharge by $22.2 \%$, within 3 months by $27.8 \%$, and at or after 1 year by $5.6 \%$.

In another survey of infection control practices related to MDR-E among 15 acute-care hospitals in Toronto, Canada, $\mathrm{CP}$ were more likely to be instituted universally for CRE patients than for ESBL-E patients for whom risk factors for transmission (eg, diarrhea, draining wounds, etc) were considered when instituting CP. For ESBL-E, CP was maintained until discharge by 5 of 15 hospitals, discontinued after 1 negative specimen by 2 of 15 hospitals, and after 3 negative specimens 1 week apart by 8 of 15 hospitals. ${ }^{77}$ For CRE, CP was maintained until discharge by 8 of 15 hospitals, discontinued after 1 negative specimen from original positive site by 1 of 15 hospitals, after 3 negative specimens 1 week apart by 4 of 15 hospitals, and was not yet determined by 2 of 15 hospitals. 


\section{Duration of Colonization}

Several studies have evaluated colonization with MDR-E longitudinally in a variety of patient populations in different countries and healthcare settings. ${ }^{21-23,78-90}$ Most studies were conducted in the acute-care setting ${ }^{21-23,79-81,83,85,87,89-91}$ and identified a population of patients infected and/or colonized with MDR-E, either ESBL-E or CRE, or MDR-Enterobacteriaceae defined by resistance to multiple classes of drugs. Patients were assessed for persistence of colonization at study-specific intervals and variable durations of follow-up. The definition of "clearance" varied between a single negative screening culture, ${ }^{79,87,90}$ at least 2 negative screening cultures, ${ }^{21,23,81,84}$ or at least 3 negative screening cultures swabs. ${ }^{80,85,91}$

ESBL-E. Among hematology-oncology patients in 5 hospitals in Germany undergoing rectal swab sampling every $10 \pm 2$ days (mean duration of hospitalization, 36.6 days), 22 of 55 patients $(40.0 \%)$ colonized with ESBL-E converted to a negative ESBL-E carrier status, and 18 of those $22(81.8 \%)$ maintained this status. ${ }^{23}$ In another university hospital cohort in Germany, with clearance defined by 3 negative cultures 1 week or more apart obtained without ESBL-directed antibiotic therapy, only 10 cases $(6.8 \%)$ were cleared from colonization. ${ }^{85}$ In a Swedish cohort in which subjects provided self-collected fecal samples, the prevalence of ESBL-E carriage was as follows: 51 of $61(84 \%)$ after 1 month, 36 of $61(66 \%)$ after 3 months, 31 of $61(55 \%)$ after 6 months, and 26 of 61 (43\%) after 12 months from a positive clinical culture. ${ }^{78}$ In a French university hospital cohort $(n=448)$ undergoing routine active surveillance on admission for previous positive culture, $40 \%$ were still colonized at first readmission; $25.6 \%$ were still colonized after 1 year, and $8.9 \%$ were still colonized after 2 years, with a median time to ESBL-E clearance (single negative rectal swab) of 6.6 months (range, 3.4-13.4 months). ${ }^{79,91}$

CRE. In a postdischarge surveillance study of tertiary-care hospital and long-term care facility patients infected or colonized with KPC-Klebsiella pneumoniae in Israel, resolution of carriage (defined as 2 consecutive negative cultures and blaKPC-PCR tests with no subsequent positive test) occurred in 65 of $125(52 \%)$ patients followed for 5 months. ${ }^{81}$ In a tertiary-hospital-based prospective cohort in Hong Kong with a median follow-up of 54 days (range, 15-421 days), the median duration of stool CRE carriage was 43 days (range, 13-119 days). ${ }^{22}$ In another tertiary-care hospital-based study in Israel, known CRE (predominantly K. pneumoniae) patients with a positive rectal screen at a subsequent hospital encounter (cases) were compared with those with a negative screen (controls). The time interval between the first positive CRE test and the next CRE surveillance test was significantly shorter among cases (median, 51 days; interquartile range [IQR], 15-131) than controls (median, 145 days; IQR, 63-287; $P=.003){ }^{89}$ In another case-control study among tertiary-care hospital patients in Israel, eradication was defined on the basis of 2 negative rectal cultures plus a negative culture from the original source of CRE isolation (eg, urine, sputum, or wounds). This screening procedure to declare presumed eradication was performed at a median of 11.9 months (IQR, 7.7-19.3) after the last positive CRE culture. The median time to CRE recurrence was 49 days (IQR, 16-130), with recurrence of the same CRE in 30 of 36 case patients. Recurrent CRE was isolated only in screening samples in 21 patients (58\%), from urine in 11 patients (31\%), and from other clinical samples in 4 patients $(11 \%){ }^{21}$

Variably Defined MDR-E. In a study involving patients with a history of military deployment hospitalized at Walter Reed National Military Medical Center, those colonized with MDR-E (defined as ESBL-producing organism or resistant to 3 of 5 antibiotic classes [ie, penicillins, cephalosporins, carbapenems, aminoglycosides, or fluoroquinolones]) underwent sequential culturing for 8 weeks or until hospital discharge. Only 1 deployed subject became decolonized over the course of the study, with a median duration of colonization of 26 days (IQR, 9-33). ${ }^{83}$ This was the only study to culture multiple anatomic sites over time; the results showed that the groin was the most sensitive anatomic site for detecting MDR-E overall but sensitivity differed by organism. In a community-based cohort in France, stool samples from adults returning after travel to tropical regions were screened for Enterobacteriaceae producing an ESBL, AmpC, and/or carbapenemase. Detection rates were $60 \%$ at 1 month, $57 \%$ at both 2 and 3 months, and $63 \%$ at both 6 and 12 months. ${ }^{86}$

\section{Patient Groups at Risk for Prolonged Colonization}

For ESBL-E, Phylogroup B2 and CTX-M-gr-9 were associated with ESBL-E carriage at 12 months in a Swedish cohort. ${ }^{78}$ In a French university hospital, having the first positive culture on screening samples only (compared to clinical \pm screening samples) was associated with ESBL-E clearance at readmission. ${ }^{79}$ Similarly, among KPC-producing CRE carriers in a university hospital in Israel, the index culture being a clinical culture (vs surveillance culture) was associated with longer mean time to negativity. ${ }^{87}$ Recurrent hospitalization between the first and last culture was also associated with a longer time to clearance of CRE colonization. This finding was also reported in a case-control study of risk factors for CRE recurrence in Israel, where a shorter time between the last positive CRE culture and presumed eradication, recurrent hospitalization, and the presence of a foreign body at the time of presumed eradication were associated with CRE recurrence. ${ }^{21}$ In another hospital-based study in Israel, persistent CRE-positive patients $(\mathrm{n}=23)$ were more often admitted from another hospital ( $59.1 \%$ vs $26.3 \% ; P=.012$ ) and were exposed to antimicrobials during the preceding 30 days, although the latter did not reach statistical significance $(57 \%$ vs $33 \%$; $P=.059){ }^{89}$ In a study of CRE carriers in a university hospital in Hong Kong that employed serial quantitative detection via fecal specimens, a higher bacterial load on initial detection of CRE, and the use of cephalosporins, carbapenems, and 
fluoroquinolones after CRE detection were associated with persistent fecal carriage. ${ }^{22}$ In a postdischarge surveillance study in Israel, resolution of CRE carriage was more likely to occur in recent acquisitions (first positive $<4$ months prior to enrollment) with 29 of 75 (39\%) remaining positive compared to remote positives (first positive $>4$ months before enrollment) with 36 of $50(72 \%)$ remaining positive $(P<.001)$. Furthermore, in remotely colonized patients, the presence of an invasive device and high Charlson score were associated with persistent carriage. ${ }^{81}$ Among adults returning to France after traveling to tropical regions and found to carry ESBL-E, CRE, or AmpC-producing Enterobacteriaceae, travelers returning from Asia had more prolonged colonization than travelers returning from sub-Saharan Africa or Latin America, and carriers of $E$. coli had a lower risk of prolonged carriage than carriers of other Enterobacteriaceae species. ${ }^{86}$

\section{Sites of Colonization}

Generally, the lower gastrointestinal tract is considered the primary site of colonization with MDR-E, though there is no universally accepted surveillance sampling strategy. The gastrointestinal tract sampling was included in nearly all of these studies. Most studies evaluating duration of colonization with MDR-E utilized stool samples, ${ }^{78,80,82,86,88}$ perirectal swab samples, ${ }^{21,79,81,84}$ or either. ${ }^{23,85}$ Original sites of infection were tested (if still relevant) in one study, ${ }^{21}$ and another study included cultures of samples obtained from multiple anatomic sites (ie, forehead, axillae, finger webs, groin, toe webs, and perirectal area). ${ }^{83}$

\section{CLOSTRIDIUM DIFFICILE}

\section{Shedding Time}

Sethi et $\mathrm{al}^{25}$ demonstrated that 1-4 weeks after conclusion of CDI treatment with vancomycin the frequencies of skin and environmental shedding increased to $58 \%$ and $50 \%$, respectively (Figure 1 ). These percentages were greater than they were at the end of CDI treatment $(32 \%$ and $14 \%$, respectively). This study suggests that skin contamination and environmental surface shedding of $C$. difficile often persist at the time of resolution of diarrhea, and recurrent shedding is common 1-4 weeks after therapy. These results provide support for the recommendation that $\mathrm{CP}$ be continued until hospital discharge if rates of CDI remain high despite implementation of standard infection control measures.

Jinno et $\mathrm{al}^{26}$ compared shedding of spores in patients with resolved CDI within the past month (the time from the end of therapy to 1 month after completion of therapy) and those with active CDI (time from diagnosis until completion of CDI treatment or until completion of 14 days of treatment in patients receiving prolonged tapering courses of vancomycin). Patients with active CDI were found to have high frequencies of positive stool, skin, and environmental cultures $(100 \%$, $63 \%$, and $51 \%$, respectively). ${ }^{26}$ Among the patients with resolved CDI, the frequency of positive stool, skin, and environmental cultures was significantly higher among patients cultured during the month after completion of treatment versus those cultured $>1$ month after treatment $(50 \%, 46 \%$, and $29 \%$ vs $18 \%, 5 \%$, and $5 \%$, respectively; $P<.01$ for each comparison). None of the patients whose CDI had resolved 6-24 months after completion of treatment had positive skin or environmental cultures. These results indicate that patients with resolved CDI may demonstrate significantly more shedding during the month following completion of treatment than those cultured $>1$ month following treatment.

Donskey et $\mathrm{al}^{92}$ showed that the likelihood of carriers having a positive skin or environmental culture or positive perirectal PCR result increased as the number of colonies per swab increased. In comparison to carriers with 1-25 colonies per swab, those with $\geq 26$ colonies per swab had significantly higher frequencies of skin shedding ( 1 of 10 [10\%] versus 10 of 15 [67\%]; $P=.012$ ) and environmental shedding ( 0 of 10 versus 9 [60\%] of $15 ; P=.003$ ) and were more likely to have positive perirectal PCR results ( 3 of 10 [30\%] versus 14 of 15 [93\%]; $P=.002$ ). The number of colonies recovered from perirectal swabs varied widely, and a low burden of colonization was associated with a low risk of skin or environmental shedding or a positive perirectal PCR result.

Shrestha et $\mathrm{al}^{93}$ and colleagues described the contamination of healthcare personnel (HCP) hands with $C$. difficile after caring for patients with resolved CDI who were no longer under CP. Patient CDI was either recent (2 days to 6 weeks after the end of CDI treatment without diarrhea) or remote (6-24 weeks after CDI treatment). Not surprisingly, the frequencies of hand contamination of HCP after contact with patients with active

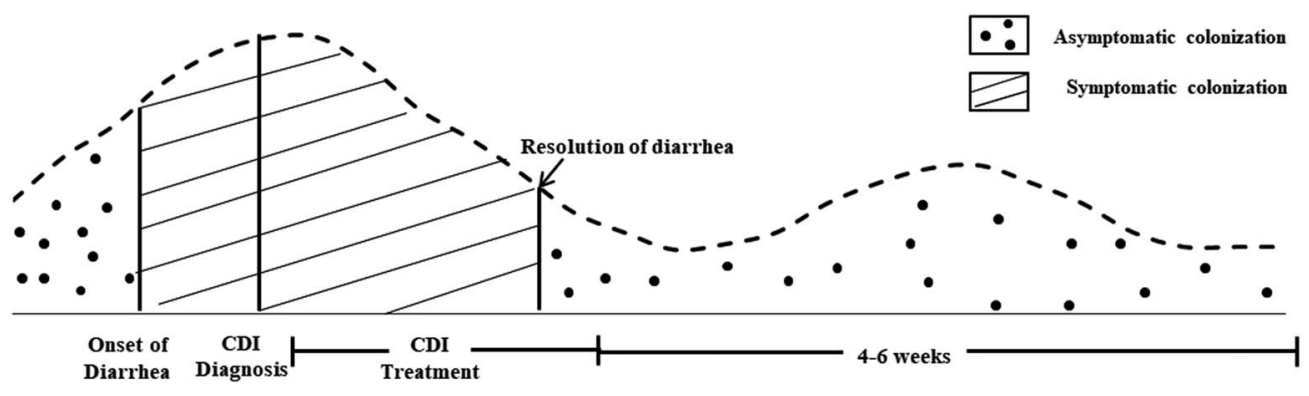

FIGURE 1. Frequency of Skin Contamination and Environmental Shedding ${ }^{116}$ 
and recent CDI were similar. Although less common, hand contamination occurred during care of patients with remote $\mathrm{CDI}$, which may support placing patients on $\mathrm{CP}$ for the duration of admission as well as re-admissions. Continuing CP after resolution of diarrhea may also reduce transmission. ${ }^{93}$

The clinical impact of $\mathrm{CP}$ based on shedding might be extrapolated from studies that investigated the effect of $\mathrm{CP}$ on asymptomatic $C$. difficile carriers. Lanzas and Dubberke ${ }^{94}$ used agent-based modeling to evaluate the potential effectiveness of methods targeting asymptomatic carriers to control C. difficile colonization and infection rates by screening patients at admission to detect asymptomatic $C$. difficile carriers and placing positive patients on CP. Scenarios simulating screening test characteristics, colonization prevalence, and type of strain present at admission were used. The investigators found on average that testing asymptomatic carriers reduced the number of new colonization cases by $40 \%-50 \%$ and hospital-onset CDI cases by $10 \%-25 \%$, compared with the baseline scenario.

A 2016 crossover experimental study showed that placing asymptomatic carriers on $\mathrm{CP}$ based on universal screening on admission significantly decreased the incidence of CDI (3 hospital-associated [HA] CDI cases per 10,000 patient days) compared to a control unit (6.9 HA-CDI per 10,000 patient days; $P<.001)$. The researchers estimate the intervention prevented approximately 63 cases. $^{27,95}$

\section{Patients with $C$. difficile Infection}

A 2009 survey of 33 acute-care hospitals in Canada identified different approaches related to the discontinuation of CP; 13 hospitals (39\%) reported that $\mathrm{CP}$ were discontinued as soon as the patients were asymptomatic, and 19 hospitals (58\%) required a period of 24-72 hours with no symptoms prior to discontinuation of CP. One hospital reported continuing $\mathrm{CP}$ until the end of the treatment for CDI; this hospital had the greatest percentage of positive test results (32\% vs $12 \%$ for all other hospitals combined; $P<.001)$. No respondent continued additional CP until discharge. ${ }^{96}$

Johnson et $\mathrm{al}^{97}$ performed a prospective, controlled study of the use of universal gloves on the incidence of CDI and C. difficile colonization. They found that the units in which the glove intervention was performed demonstrated a decrease of C. difficile incidence from 7.7 to 1.5 cases per 1,000 patient discharges $(P=.015)$. Contrary to this finding, the control units without the intervention had unchanged incidence in the intervention and control phases. Colonization rates also decreased in the units undergoing the interventions $(17 \%$ vs $9 \% ; P=.029)$. This study suggests that the use of vinyl gloves may significantly reduce the transmission of $C$. difficile diarrhea and therefore should be used for the duration of hospitalization. ${ }^{97}$

\section{USE OF MOLECULAR TESTING TO DETERMINE DURATION OF CP}

Various methods and procedures have been used to screen patients for MRSA, VRE, ESBL, and CRE colonization. The simplest approach is to culture swab specimens directly on solid agar media. Alternatively, sensitivity can be enhanced by first placing swabs in enrichment broth. Following incubation, broth is inoculated on to agar plates. Broth incubation enhances recovery of MRSA, ${ }^{98}$ but lengthens laboratory turnaround time of results.

\section{Comparison of Molecular and Standard Methods on Sensitivity, Specificity, and Duration of Positivity}

The diagnostic advantage of molecular methods (eg, PCR) is enhanced sensitivity compared to culture. Unfortunately, scientific documentation of this phenomenon has been challenged using weak study methodology. The most common methodology involves performing PCR in parallel with culture, with the latter designated as the gold standard. PCR-positive/culture-negative specimens are then retested with an "arbiter" PCR. If positive, the specimen is assigned positive screen status. ${ }^{99-114}$ This methodology (called "partial verification") introduces bias in favor of PCR. ${ }^{115}$ For MRSA, Yam et $\mathrm{al}^{99}$ tested 1,246 nares swab specimens with the Lightcycler MRSA Advanced (Roche Molecular Diagnostics, Basel Switzerland), a laboratory developed test (LDT) PCR and, in parallel, cultured all specimens. The gold standard was defined as positive culture or positivity by both molecular assays. A preferable method may be to perform multiple PCR assays with similar sensitivity on all specimens using a "consensus" gold standard definition. The sensitivities of culture, Lightcycler MRSA Advanced, and the LDT were $84 \%, 83 \%$, and $76 \%$, respectively, with no apparent statistical significance. ${ }^{99}$ No similar studies were available for VRE or CRE assays.

Recently, FDA-cleared molecular methods (eg, PCR) for detecting MRSA, VRE, and CRE have become commercially available in the United States. For MRSA, commonly used assays include the Xpert MRSA XT and Xpert SA Nasal Complete (Cepheid, Sunnyvale, CA); BD MAX MRSA and BD MAX StaphSR (Becton Dickinson, Franklin Lakes, NJ); Lightcycler MRSA Advanced (Roche Molecular Diagnostics, Basel Switzerland); and the Cobas MRSA/SA Test (Roche Molecular Diagnostics). The Xpert VanA assay (Cepheid) has been cleared by the FDA to detect VRE mediated by vanA, and the Xpert CarbaR (Cepheid) detects various determinants of CRE including blaKPC, blaNDM, blaVIM, blaIMP-1 and blaOXA-48 (including OXA-48-like variants OXA-181 and OXA-232). At this time, no molecular assays that detect ESBL determinants have been cleared by the FDA.

\section{DEVELOPING POLICIES FOR \\ DISCONTINUATION OF CONTACT PRECAUTIONS}

Policies dictating the duration of contact precautions can provide guidance to clinicians and infection prevention and control programs in acute-care hospitals. Given the limited data on the duration of organism colonization and the impact 
TA в LE 2. Potential Components for Inclusion in a Policy for Duration of Contact Precautions With Examples

\begin{tabular}{ll}
\hline Components & Examples \\
\hline Inclusion criteria & $\begin{array}{c}\text { Elapsed time since last infection with organism until consideration for discontinuation of CP } \\
\text { (ie, 3, 6, 12 months) } \\
\text { Concomitant antibiotic use within a specified period (ie, 24-48 hours prior to sampling), } \\
\text { active infection, hospitalization in an outbreak, or hyperendemic period at the facility }\end{array}$ \\
$\begin{array}{l}\text { No. of surveillance samples } \\
\text { Surveillance sample source }\end{array}$ & $\begin{array}{l}\text { Nares (MRSA), perirectal (VRE, MDR-E), stool (VRE, MDR-GNR) } \\
\text { Surveillance sample frequency } \\
\text { Testing methodology }\end{array}$ \\
$\begin{array}{l}\text { Final arbiter for discontinuation of CP } \\
\text { Policy implementation strategy }\end{array}$ & $\begin{array}{l}\text { Bealthcare epidemiologist, infection preventionist, other hospital/ID leadership } \\
\text { Clinical staff with assistance from the Infection Prevention and Control Program vs active } \\
\text { monitoring and implementation led by the Infection Control and Prevention Program }\end{array}$
\end{tabular}

NOTE. CP, contact precautions; MRSA, methicillin-resistant Staphylococcus aureus; VRE, vancomycin-resistant enterococci (VRE); PCR, polymerase chain reaction; ID, infectious diseases; MDR-GNR, multidrug-resistant Gram-negative rods; CRE, carbapenem-resistant Enterobacteriaceae; ESBL, extended-spectrum $\beta$-lactamase

of $\mathrm{CP}$ in reducing spread of organisms in the healthcare environment, no universally recommended approach exists for making decisions regarding CP duration or discontinuation for any epidemiologically significant organism. However, hospital policies addressing duration of CP should include several components (see Table 2 ). In adopting a policy to guide decisions regarding discontinuation of $\mathrm{CP}$, hospitals should carefully assess their institutional risks, priorities, and resources. Infection prevention and control leadership should revisit and revise policies if the epidemiology of specific organisms of concern change, particularly in an outbreak or hyperendemic situation. Additional factors, such as the cost and feasibility of implementation, should be considered in adopting a policy for CP discontinuation.

\section{CONCLUSIONS AND AREAS FOR FUTURE RESEARCH}

Data guiding decisions regarding the duration of CP are generally limited to studies that often describe the duration of low-level organism colonization rather than interventional studies evaluating the clinical impact of discontinuing CP in different settings. Extrapolation of published data is limited based on variability in definitions of colonization and an inadequate understanding of the impact of $\mathrm{CP}$ on preventing organism transmission. Further research is needed to improve our understanding of the role of molecular testing in evaluating the transmissibility of organisms in healthcare settings, and how these tests perform in comparison to culture-based methodologies in guiding decisions regarding the duration of CP. Research studies should be performed in "real-world" settings to establish a stronger evidence base on which to optimize the use of CP in reducing the spread of MDROs and $C$. difficile. Large, multicenter, prospective cluster-randomized trials using appropriate outcomes and time spans to evaluate these outcomes would be valuable in the development of evidence-based guidelines for the implementation of $\mathrm{CP}$, including decisions regarding the duration of CP in acute-care hospitals.

\section{ACKNOWLEDGMENTS}

This study was supported in part by the SHEA Research Network (SRN). We express immense gratitude to Valerie M. Deloney, MBA for her editorial assistance and invaluable organizational expertise in coordinating this project. We also wish to thank Erica S. Shenoy, MD, PhD, for her extensive and thoughtful review of the manuscript.

The following disclosures are a reflection of what has been reported to SHEA. To provide thorough transparency, SHEA requires full disclosure of all relationships, regardless of relevancy to the guidance topic. Evaluation of such relationships as potential conflicts of interest is determined by a review process which includes assessment by the SHEA Conflict of Interest Committee. The assessment of disclosed relationships for possible conflicts of interest is based on the relative weight of the financial relationship (ie, monetary amount) and the relevance of the relationship (ie, the degree to which an association might reasonably be interpreted by an independent observer as related to the topic or recommendation of consideration). Readers of this guidance should be mindful of this when reviewing the list of disclosures.

D.B., G.B., K.P., M.B., and S.L. report that they have nothing to disclose. D.M. Springer Nature for book and journal editing and research grants from NIH, AHRQ, VA HSRD and CDC. R.M. reports a research grant/contract with Medimmune and Johns Hopkins (lead in multicenter trial: HAIs Among Surgical and ICU Patients: Implications for Interventions and Treatment). L.S.M.P. reports an advisory/consultant role with Xenex/Clorox, and speaker honorarium from Ecolab. K.V.S. reports research grants/contracts with Nanosphere, Inc. (Performance of the Verigene Gram-Negative Blood Culture Test [BC-GN] in Pediatric Patients), Techlab, The Children's Hospital of Philadelphia (Evaluation of the Performance of the Shiga Toxin Quik Chek), and Premier EHEC and CHROMagar 0157 (The Detection of EHEC Infections in Children). J.H. reports an advisory/consultant role with Gilead. T.W. reports an advisory/consultant role and research grant with Clorox Healthcare (What is the Role of Improved Hydrogen Peroxide in the Operating Room?) and research grants/contracts with the CDC (Enhancing Surveillance Among Refugees), Pfizer (Understanding Why Patients Accept Vaccines: Socio-Behavioral Approach), and University of Louisville research grants $>\$ 25,000$ with Pfizer and Clorox Healthcare.

Address correspondence to David Banach, MD, MPH, MS, University of Connecticut Health Center, 263 Farmington Ave, Farmington, CT 06030 (dbanach@uchc.edu). 


\section{REFERENCES}

1. Siegel JD, Rhinehart E, Jackson M, Chiarello L, HICPAC. 2007 Guideline for isolation precautions: preventing transmission of infectious agents in health care settings. Am J Infect Control 2007;35:S65-S164.

2. Dashiell-Earp CN, Bell DS, Ang AO, Uslan DZ. Do physicians spend less time with patients in contact isolation? A time-motion study of internal medicine interns. JAMA Intern Med 2014;174: 814-815.

3. Harris AD, Pineles L, Belton B, et al. Universal glove and gown use and acquisition of antibiotic-resistant bacteria in the ICU: a randomized trial. JAMA 2013;310:1571-1580.

4. Masse V, Valiquette L, Boukhoudmi S, et al. Impact of methicillin-resistant Staphylococcus aureus contact isolation units on medical care. PLoS One 2013;8:e57057.

5. Morgan DJ, Murthy R, Munoz-Price LS, et al. Reconsidering contact precautions for endemic methicillin-resistant Staphylococcus aureus and vancomycin-resistant Enterococcus. Infect Control Hosp Epidemiol 2015;36:1163-1172.

6. Martin EM, Russell D, Rubin Z, et al. Elimination of routine contact precautions for endemic methicillin-resistant Staphylococcus aureus and vancomycin-resistant Enterococcus: a retrospective quasi-experimental study. Infect Control Hosp Epidemiol 2016;37:1323-1330.

7. Edmond MB, Masroor N, Stevens MP, Ober J, Bearman G. The impact of discontinuing contact precautions for VRE and MRSA on device-associated infections. Infect Control Hosp Epidemiol 2015;36:978-980.

8. Almyroudis NG, Osawa R, Samonis G, et al. Discontinuation of systematic surveillance and contact precautions for vancomycinresistant Enterococcus (VRE) and its impact on the incidence of VRE faecium bacteremia in patients with hematologic malignancies. Infect Control Hosp Epidemiol 2016;37:398-403.

9. Russell D, Beekmann SE, Polgreen PM, Rubin Z, Uslan DZ. Routine use of contact precautions for methicillin-resistant Staphylococcus aureus and vancomycin-resistant Enterococcus: Which way is the pendulum swinging? Infect Control Hosp Epidemiol 2016;37:36-40.

10. Society for Healthcare Epidemiology of America. Handbook for SHEA-Sponsored Guidelines and Expert Guidance Documents. SHEA website. http://www.shea-online.org/images/docs/2017_ Handbook.pdf. Published 2015. Accessed January 2017.

11. Shenoy ES, Hsu H, Noubary F, Hooper DC, Walensky RP. National survey of infection preventionists: policies for discontinuation of contact precautions for methicillin-resistant Staphylococcus aureus and vancomycin-resistant enterococcus. Infect Control Hosp Epidemiol 2012;33:1272-1275.

12. Huckabee CM, Huskins WC, Murray PR. Predicting clearance of colonization with vancomycin-resistant Enterococci and methicillin-resistant Staphylococcus aureus by use of weekly surveillance cultures. J Clin Microbiol 2009;47:1229-1230.

13. Scanvic A, Denic L, Gaillon S, Giry P, Andremont A, Lucet JC. Duration of colonization by methicillin-resistant Staphylococcus aureus after hospital discharge and risk factors for prolonged carriage. Clin Infect Dis 2001;32:1393-1398.

14. Baden LR, Thiemke W, Skolnik A, et al. Prolonged colonization with vancomycin-resistant Enterococcus faecium in long-term care patients and the significance of "clearance. Clin Infect Dis 2001;33:1654-1660.
15. Donskey CJ, Hoyen CK, Das SM, Helfand MS, Hecker MT. Recurrence of vancomycin-resistant Enterococcus stool colonization during antibiotic therapy. Infect Control Hosp Epidemiol 2002;23:436-40.

16. Patel R, Allen SL, Manahan JM, et al. Natural history of vancomycin-resistant enterococcal colonization in liver and kidney transplant recipients. Liver Transpl 2001;7:27-31.

17. Sohn KM, Peck KR, Joo EJ, et al. Duration of colonization and risk factors for prolonged carriage of vancomycin-resistant enterococci after discharge from the hospital. Int J Infect Dis 2013; 17:e240-e246.

18. Henning KJ, Delencastre H, Eagan J, et al. Vancomycin-resistant Enterococcus faecium on a pediatric oncology ward: duration of stool shedding and incidence of clinical infection. Pediatr Infect Dis J 1996;15:848-854.

19. Karki S, Land G, Aitchison S, et al. Long-term carriage of vancomycin-resistant enterococci in patients discharged from hospitals: a 12-year retrospective cohort study. J Clin Microbiol 2013;51:3374-3379.

20. Byers KE, Anglim AM, Anneski CJ, Farr BM. Duration of colonization with vancomycin-resistant Enterococcus. Infect Control Hosp Epidemiol 2002;23:207-211.

21. Bart Y, Paul M, Eluk O, Geffen Y, Rabino G, Hussein K. Risk factors for recurrence of carbapenem-resistant Enterobacteriaceae carriage: case-control study. Infect Control Hosp Epidemiol 2015;36: 936-941.

22. Cheng VC, Chan JF, Wong SC, et al. Proactive infection control measures to prevent nosocomial transmission of carbapenemresistant Enterobacteriaceae in a non-endemic area. Chin Med J (Engl) 2013;126:4504-4509.

23. Vehreschild MJ, Hamprecht A, Peterson L, et al. A multicentre cohort study on colonization and infection with ESBL-producing Enterobacteriaceae in high-risk patients with haematological malignancies. J Antimicrob Chemother 2014;69:3387-3392.

24. Yokoe DS, Anderson DJ, Berenholtz SM, et al. A compendium of strategies to prevent healthcare-associated infections in acute care hospitals: 2014 updates. Infect Control Hosp Epidemiol 2014;35: 967-977.

25. Sethi AK, Al-Nassir WN, Nerandzic MM, Bobulsky GS, Donskey CJ. Persistence of skin contamination and environmental shedding of Clostridium difficile during and after treatment of $C$. difficile infection. Infect Control Hosp Epidemiol 2010;31:21-27.

26. Jinno S, Kundrapu S, Guerrero DM, Jury LA, Nerandzic MM, Donskey CJ. Potential for transmission of Clostridium difficile by asymptomatic acute care patients and long-term care facility residents with prior $C$. difficile infection. Infect Control Hosp Epidemiol 2012;33:638-639.

27. Longtin Y, Paquet-Bolduc B, Gilca R, et al. Effect of detecting and isolating Clostridium difficile carriers at hospital admission on the incidence of $C$. difficile infections: a quasi-experimental controlled study. JAMA Intern Med 2016;176:796-804.

28. Health Facilities and Regulation: (210 ILCS 83/) MRSA Screening and Reporting Act, PA 95-312, eff. 8/20/07 (2007).

29. Medical Facility Infection Control and Prevention Act (Niles Law) Senate Bill No. 1058, Chapter 296, (2008).

30. Calfee DP, Salgado CD, Milstone AM, et al. Strategies to prevent methicillin-resistant Staphylococcus aureus transmission and infection in acute care hospitals: 2014 update. Infect Control Hosp Epidemiol 2014;35:S108-S132. 
31. Kluytmans J, van Belkum A, Verbrugh H. Nasal carriage of Staphylococcus aureus: epidemiology, underlying mechanisms, and associated risks. Clin Microbiol Rev 1997;10:505-520.

32. Shenoy ES, Walensky RP, Lee H, Orcutt B, Hooper DC. Resource burden associated with contact precautions for methicillinresistant Staphylococcus aureus and vancomycin-resistant Enterococcus: the patient access managers' perspective. Infect Control Hosp Epidemiol 2012;33:849-852.

33. Siegel JD, Rhinehart E, Jackson M, Chiarello L, Committee HICPA. Management of multidrug-resistant organisms in health care settings, 2006. Am J Infect Control 2007;35:S165-S193.

34. Moody J, Septimus E, Hickok J, et al. Infection prevention practices in adult intensive care units in a large community hospital system after implementing strategies to reduce health care-associated, methicillin-resistant Staphylococcus aureus infections. Am J Infect Control 2013;41:126-130.

35. Morgan DJ, Pineles L, Shardell M, et al. The effect of contact precautions on healthcare worker activity in acute care hospitals. Infect Control Hosp Epidemiol 2013;34:69-73.

36. Shenoy ES, Kim J, Rosenberg ES, et al. Discontinuation of contact precautions for methicillin-resistant Staphylococcus aureus: a randomized controlled trial comparing passive and active screening with culture and polymerase chain reaction. Clin Infect Dis 2013;57:176-84.

37. Shenoy ES, Lee H, Cotter JA, et al. Impact of rapid screening for discontinuation of methicillin-resistant Staphylococcus aureus contact precautions. Am J Infect Control 2016;44:215-221.

38. Gase KA, Reese SM. Journal club: impact of rapid screening for discontinuation of methicillin-resistant Staphylococcus aureus contact precautions. Am J Infect Control 2016;44:475-476.

39. Vikram HR, Dumigan DG, Kohan C, Havill NL, Tauman A, Boyce JM. Discontinuation of contact precautions for patients no longer colonized with methicillin-resistant Staphylococcus aureus. Infect Control Hosp Epidemiol 2010;31:541-543.

40. Goldsack JC, DeRitter C, Power M, et al. Clinical, patient experience and cost impacts of performing active surveillance on known methicillin-resistant Staphylococcus aureus positive patients admitted to medical-surgical units. Am J Infect Control 2014;42:1039-1043.

41. Quan KA, Cousins SM, Porter DD, Puppo RA, Huang SS. Automated tracking and ordering of precautions for multidrugresistant organisms. Am J Infect Control 2015;43:577-580.

42. Carson PJ, Danford M, Carson G, et al. "Bringing down the flag": removing the contact precautions label for non-hospitalized patients with prior methicillin-resistant Staphylococcus aureus infection or colonization. Infect Control Hosp Epidemiol 2015;36:578-580.

43. Robicsek A, Beaumont JL, Peterson LR. Duration of colonization with methicillin-resistant Staphylococcus aureus. Clin Infect Dis 2009;48:910-913.

44. Sanford MD, Widmer AF, Bale MJ, Jones RN, Wenzel RP. Efficient detection and long-term persistence of the carriage of methicillin-resistant Staphylococcus aureus. Clin Infect Dis 1994;19:1123-1128.

45. Datta R, Huang SS. Risk of infection and death due to methicillinresistant Staphylococcus aureus in long-term carriers. Clin Infect Dis 2008;47:176-181.

46. Marschall J, Mühlemann K. Duration of methicillin-resistant Staphylococcus aureus carriage, according to risk factors for acquisition. Infect Control Hosp Epidemiol 2006;27:1206-1212.
47. Lucet JC, Paoletti X, Demontpion C, et al. Carriage of methicillinresistant Staphylococcus aureus in home care settings: prevalence, duration, and transmission to household members. Arch Intern Med 2009;169:1372-1378.

48. Fritz SA, Krauss MJ, Epplin EK, et al. The natural history of contemporary Staphylococcus aureus nasal colonization in community children. Pediatr Infect Dis J 2011;30:349-351.

49. Cluzet VC, Gerber JS, Nachamkin I, et al. Duration of colonization and determinants of earlier clearance of colonization with methicillin-resistant Staphylococcus aureus. Clin Infect Dis 2015;60:1489-1496.

50. Rogers C, Sharma A, Rimland D, et al. Duration of colonization with methicillin-resistant Staphylococcus aureus in an acute care facility: a study to assess epidemiologic features. Am J Infect Control 2014;42:249-253.

51. Gibson KE, McNamara SE, Cassone M, et al. Methicillin-resistant Staphylococcus aureus: site of acquisition and strain variation in high-risk nursing home residents with indwelling devices. Infect Control Hosp Epidemiol 2014;35:1458-1465.

52. Bradley SF, Terpenning MS, Ramsey MA, et al. Methicillinresistant Staphylococcus aureus: colonization and infection in a long-term care facility. Ann Intern Med 1991;115:417-422.

53. Manzur A, Gudiol F. Methicillin-resistant Staphylococcus aureus in long-term-care facilities. Clin Microbiol Infect 2009;15:26-30.

54. Pacio GA, Visintainer P, Maguire G, Wormser GP, Raffalli J, Montecalvo MA. Natural history of colonization with vancomycinresistant enterococci, methicillin-resistant Staphylococcus aureus, and resistant Gram-negative bacilli among long-term-care facility residents. Infect Control Hosp Epidemiol 2003;24:246-250.

55. Muder RR, Brennen C, Wagener MM, et al. Methicillin-resistant staphylococcal colonization and infection in a long-term care facility. Ann Intern Med 1991;114:107-112.

56. Lautenbach E, Tolomeo P, Nachamkin I, Hu B, Zaoutis TE. The impact of household transmission on duration of outpatient colonization with methicillin-resistant Staphylococcus aureus. Epidemiol Infect 2010;138:683-685.

57. Larsson AK, Gustafsson E, Nilsson AC, Odenholt I, Ringberg H, Melander E. Duration of methicillin-resistant Staphylococcus aureus colonization after diagnosis: a four-year experience from southern Sweden. Scand J Infect Dis 2011;43:456-462.

58. Ridenour GA, Wong ES, Call MA, Climo MW. Duration of colonization with methicillin-resistant Staphylococcus aureus among patients in the intensive care unit: implications for intervention. Infect Control Hosp Epidemiol 2006;27:271-278.

59. Mattner F, Biertz F, Ziesing S, Gastmeier P, Chaberny IF. Longterm persistence of MRSA in re-admitted patients. Infection. 2010;38:363-371.

60. Ghosh A, Jiao L, Al-Mutawa F, O'Neill C, Mertz D, Team Hamilton HSIPaC. Value of an active surveillance policy to document clearance of meticillin-resistant Staphylococcus aureus and vancomycin-resistant enterococci amongst inpatients with prolonged admissions. J Hosp Infect 2014;88:230-233.

61. Cluzet VC, Gerber JS, Nachamkin I, et al. Risk factors for recurrent colonization with methicillin-resistant Staphylococcus aureus in community-dwelling adults and children. Infect Control Hosp Epidemiol 2015;36:786-793.

62. Couderc C, Thiébaut AC, Lawrence C, et al. Fluoroquinolone impact on nasal methicillin-resistant and methicillin-sensitive Staphylococcus aureus colonization durations in neurologic long-termcare facilities. Antimicrob Agents Chemother 2015;59:7621-7628. 
63. Shenoy ES, Paras ML, Noubary F, Walensky RP, Hooper DC. Natural history of colonization with methicillin-resistant Staphylococcus aureus (MRSA) and vancomycin-resistant Enterococcus (VRE): a systematic review. BMC Infect Dis 2014;14:177.

64. McKinnell JA, Huang SS, Eells SJ, Cui E, Miller LG. Quantifying the impact of extranasal testing of body sites for methicillinresistant Staphylococcus aureus colonization at the time of hospital or intensive care unit admission. Infect Control Hosp Epidemiol 2013;34:161-170.

65. Recommendations for preventing the spread of vancomycin resistance. Recommendations of the Hospital Infection Control Practices Advisory Committee (HICPAC). MMWR Recomm Rep 1995;44:1-13.

66. Henard S, Lozniewski A, Aissa N, Jouzeau N, Rabaud C. Evaluation of the duration of vanA vancomycin-resistant Enterococcus faecium carriage and clearance during a large-scale outbreak in a region of eastern France. Am J Infect Control 2011;39:169-171.

67. Huang SS, Rifas-Shiman SL, Pottinger JM, et al. Improving the assessment of vancomycin-resistant enterococci by routine screening. J Infect Dis 2007;195:339-346.

68. Goetz AM, Rihs JD, Wagener MM, Muder RR. Infection and colonization with vancomycin-resistant Enterococcus faecium in an acute care Veterans Affairs Medical Center: a 2-year survey. Am J Infect Control 1998;26:558-562.

69. Singh J, Esparza S, Patterson M, Vogel K, Patel B, Gornick W. Vancomycin-resistant Enterococcus in pediatric oncology patients: balancing infection prevention and family-centered care. J Pediatr Hematol Oncol 2013;35:227-231.

70. Yoon YK, Lee SE, Lee J, et al. Risk factors for prolonged carriage of vancomycin-resistant Enterococcus faecium among patients in intensive care units: a case-control study. J Antimicrob Chemother 2011;66:1831-1838.

71. Zhou Q, Moore C, Eden S, Tong A, McGeer A, Team MSHIC. Factors associated with acquisition of vancomycin-resistant enterococci (VRE) in roommate contacts of patients colonized or infected with VRE in a tertiary care hospital. Infect Control Hosp Epidemiol 2008;29:398-403.

72. Beezhold DW, Slaughter S, Hayden MK, et al. Skin colonization with vancomycin-resistant enterococci among hospitalized patients with bacteremia. Clin Infect Dis 1997;24:704-706.

73. Haverkate MR, Derde LP, Brun-Buisson C, Bonten MJ, Bootsma MC. Duration of colonization with antimicrobial-resistant bacteria after ICU discharge. Intensive Care Med 2014;40:564-571.

74. Weinstein JW, Tallapragada S, Farrel P, Dembry LM. Comparison of rectal and perirectal swabs for detection of colonization with vancomycin-resistant enterococci. J Clin Microbiol 1996;34:210-212.

75. NCEZID, DHQP, CDC. Guidance for Control of Carbapenemresistant Enterobacteriaceae (CRE) Toolkit. Centers for Disease Control and Prevention website. https://www.cdc.gov/hai/ organisms/cre/cre-toolkit/index.html. Published 2012. Accessed July 2016.

76. Drees M, Pineles L, Harris AD, Morgan DJ. Variation in definitions and isolation procedures for multidrug-resistant Gram-negative bacteria: a survey of the Society for Healthcare Epidemiology of America Research Network. Infect Control Hosp Epidemiol 2014;35:362-366.

77. Lowe CF, Katz K, McGeer AJ, Muller MP, Group TEW. Efficacy of admission screening for extended-spectrum beta-lactamase producing Enterobacteriaceae. PLoS One 2013;8:e62678.
78. Titelman E, Hasan CM, Iversen A, et al. Faecal carriage of extended-spectrum $\beta$-lactamase-producing Enterobacteriaceae is common 12 months after infection and is related to strain factors. Clin Microbiol Infect 2014;20:O508-O515.

79. Birgand G, Armand-Lefevre L, Lolom I, Ruppe E, Andremont A, Lucet JC. Duration of colonization by extended-spectrum $\beta$-lactamase-producing Enterobacteriaceae after hospital discharge. Am J Infect Control 2013;41:443-447.

80. Löhr IH, Rettedal S, Natås OB, Naseer U, Oymar K, Sundsfjord A. Long-term faecal carriage in infants and intra-household transmission of CTX-M-15-producing Klebsiella pneumoniae following a nosocomial outbreak. J Antimicrob Chemother 2013;68: 1043-1048.

81. Feldman N, Adler A, Molshatzki N, et al. Gastrointestinal colonization by KPC-producing Klebsiella pneumoniae following hospital discharge: duration of carriage and risk factors for persistent carriage. Clin Microbiol Infect 2013;19:E190-E196.

82. Tham J, Walder M, Melander E, Odenholt I. Duration of colonization with extended-spectrum beta-lactamase-producing Escherichia coli in patients with travellers' diarrhoea. Scand J Infect Dis 2012;44:573-577.

83. Weintrob AC, Roediger MP, Barber M, et al. Natural history of colonization with Gram-negative multidrug-resistant organisms among hospitalized patients. Infect Control Hosp Epidemiol 2010; 31:330-337.

84. O'Fallon E, Gautam S, D'Agata EM. Colonization with multidrug-resistant Gram-negative bacteria: prolonged duration and frequent cocolonization. Clin Infect Dis 2009;48: 1375-1381.

85. Kola A, Holst M, Chaberny IF, Ziesing S, Suerbaum S, Gastmeier P. Surveillance of extended-spectrum beta-lactamase-producing bacteria and routine use of contact isolation: experience from a three-year period. J Hosp Infect 2007;66:46-51.

86. Ruppé E, Armand-Lefêvre L, Estellat C, et al. High rate of acquisition but short duration of carriage of multidrug-resistant Enterobacteriaceae after travel to the tropics. Clin Infect Dis 2015;61:593-600.

87. Zimmerman FS, Assous MV, Bdolah-Abram T, Lachish T, Yinnon AM, Wiener-Well Y. Duration of carriage of carbapenem-resistant Enterobacteriaceae following hospital discharge. Am J Infect Control 2013;41:190-194.

88. Tandé D, Boisramé-Gastrin S, Münck MR, et al. Intrafamilial transmission of extended-spectrum-beta-lactamase-producing Escherichia coli and Salmonella enterica Babelsberg among the families of internationally adopted children. J Antimicrob Chemother 2010;65:859-865.

89. Schechner V, Kotlovsky T, Tarabeia J, et al. Predictors of rectal carriage of carbapenem-resistant Enterobacteriaceae (CRE) among patients with known CRE carriage at their next hospital encounter. Infect Control Hosp Epidemiol 2011;32: 497-503.

90. Lewis JD, Enfield KB, Mathers AJ, Giannetta ET, Sifri CD. The limits of serial surveillance cultures in predicting clearance of colonization with carbapenemase-producing Enterobacteriaceae. Infect Control Hosp Epidemiol 2015;36:835-837.

91. Kim NH, Han WD, Song KH, et al. Successful containment of carbapenem-resistant Enterobacteriaceae by strict contact precautions without active surveillance. Am J Infect Control 2014; 42:1270-1273. 
92. Donskey CJ, Sunkesula VC, Jencson AL, et al. Utility of a commercial PCR assay and a clinical prediction rule for detection of toxigenic Clostridium difficile in asymptomatic carriers. J Clin Microbiol 2014;52:315-318.

93. Shrestha SK, Sunkesula VC, Kundrapu S, Tomas ME, Nerandzic MM, Donskey CJ. Acquisition of Clostridium difficile on hands of healthcare personnel caring for patients with resolved C. difficile infection. Infect Control Hosp Epidemiol 2016;37:475-477.

94. Lanzas C, Dubberke ER. Effectiveness of screening hospital admissions to detect asymptomatic carriers of Clostridium difficile: a modeling evaluation. Infect Control Hosp Epidemiol 2014;35:1043-1050.

95. Longtin Y, Gilca R, Loo VG. Effect of detecting and isolating asymptomatic Clostridium difficile carriers-reply. JAMA Intern Med 2016;176:1573.

96. Gravel D, Gardam M, Taylor G, et al. Infection control practices related to Clostridium difficile infection in acute care hospitals in Canada. Am J Infect Control 2009;37:9-14.

97. Johnson S, Gerding DN, Olson MM, et al. Prospective, controlled study of vinyl glove use to interrupt Clostridium difficile nosocomial transmission. Am J Med 1990;88:137-140.

98. Wolk DM, Picton E, Johnson D, et al. Multicenter evaluation of the Cepheid Xpert methicillin-resistant Staphylococcus aureus (MRSA) test as a rapid screening method for detection of MRSA in nares. J Clin Microbiol 2009;47:758-764.

99. Yam WC, Siu GK, Ho PL, et al. Evaluation of the LightCycler methicillin-resistant Staphylococcus aureus (MRSA) advanced test for detection of MRSA nasal colonization. J Clin Microbiol 2013;51:2869-2874.

100. Lepainteur M, Delattre S, Cozza S, Lawrence C, Roux AL, Rottman M. Comparative evaluation of two PCR-based methods for detection of methicillin-resistant Staphylococcus aureus (MRSA): Xpert MRSA Gen 3 and BD-Max MRSA XT. J Clin Microbiol 2015;53:1955-1958.

101. Patel PA, Robicsek A, Grayes A, et al. Evaluation of multiple real-time PCR tests on nasal samples in a large MRSA surveillance program. Am J Clin Pathol 2015;143:652-658.

102. Kim MH, Lee WI, Kang SY. Detection of methicillin-resistant Staphylococcus aureus in healthcare workers using real-time polymerase chain reaction. Yonsei Med J 2013;54:1282-1284.

103. Lee S, Park YJ, Park KG, et al. Comparative evaluation of three chromogenic media combined with broth enrichment and the real-time PCR-based Xpert MRSA assay for screening of methicillin-resistant Staphylococcus aureus in nasal swabs. Ann Lab Med 2013;33:255-260.

104. Huh HJ, Kim ES, Chae SL. Methicillin-resistant Staphylococcus aureus in nasal surveillance swabs at an intensive care unit: an evaluation of the LightCycler MRSA advanced test. Ann Lab Med 2012;32:407-412.

105. Dalpke AH, Hofko M, Zimmermann S. Comparison of the BD Max methicillin-resistant Staphylococcus aureus (MRSA) assay and the BD GeneOhm MRSA achromopeptidase assay with direct- and enriched-culture techniques using clinical specimens for detection of MRSA. J Clin Microbiol 2012;50:3365-3367.

106. Arcenas RC, Spadoni S, Mohammad A, et al. Multicenter evaluation of the LightCycler MRSA advanced test, the Xpert MRSA Assay, and MRSASelect directly plated culture with simulated workflow comparison for the detection of methicillin-resistant Staphylococcus aureus in nasal swabs. J Mol Diagn 2012;14:367-375.

107. Roisin S, Laurent C, Nonhoff C, et al. Positive predictive value of the Xpert MRSA assay diagnostic for universal patient screening at hospital admission: influence of the local ecology. Eur J Clin Microbiol Infect Dis 2012;31:873-880.

108. Hombach M, Pfyffer GE, Roos M, Lucke K. Detection of methicillin-resistant Staphylococcus aureus (MRSA) in specimens from various body sites: performance characteristics of the BD GeneOhm MRSA assay, the Xpert MRSA assay, and broth-enriched culture in an area with a low prevalence of MRSA infections. J Clin Microbiol 2010;48:3882-3887.

109. Peterson LR, Liesenfeld O, Woods CW, et al. Multicenter evaluation of the LightCycler methicillin-resistant Staphylococcus aureus (MRSA) advanced test as a rapid method for detection of MRSA in nasal surveillance swabs. J Clin Microbiol 2010;48:1661-1666.

110. Nulens E, Descheemaeker P, Deurenberg RH, Stobberingh EE, Gordts B. Contribution of two molecular assays as compared to selective culture for MRSA screening in a low MRSA prevalence population. Infection 2010;38:98-101.

111. Wolk DM, Marx JL, Dominguez L, Driscoll D, Schifman RB. Comparison of MRSASelect agar, CHROMagar methicillinresistant Staphylococcus aureus (MRSA) medium, and Xpert MRSA PCR for detection of MRSA in nares: diagnostic accuracy for surveillance samples with various bacterial densities. J Clin Microbiol 2009;47:3933-3936.

112. Rossney AS, Herra CM, Brennan GI, Morgan PM, O'Connell B. Evaluation of the Xpert methicillin-resistant Staphylococcus aureus (MRSA) assay using the GeneXpert real-time PCR platform for rapid detection of MRSA from screening specimens. J Clin Microbiol 2008;46:3285-3290.

113. Silbert S, Kubasek C, Galambo F, Vendrone E, Widen R. Evaluation of BD Max StaphSR and BD Max MRSAXT assays using ESwab-collected specimens. J Clin Microbiol 2015;53: 2525-2529.

114. Babady NE, Gilhuley K, Cianciminio-Bordelon D, Tang YW. Performance characteristics of the Cepheid Xpert vanA assay for rapid identification of patients at high risk for carriage of vancomycinresistant Enterococci. J Clin Microbiol 2012;50:3659-3663.

115. Editorial and Publishing Policy Resource: Cochrane Handbook for Diagnostic Test Accuracy Reviews 2009, Chapter 9. Assessing methodological quality. Cochrane Collaboration website. http:// methods.cochrane.org/sdt/sites/methods.cochrane.org.sdt/files/ uploads/ch09_Oct09.pdf. Accessed July 10, 2016.

116. Donskey CJ, Kundrapu S, Deshpande A. Colonization versus carriage of Clostridium difficile. Infect Dis Clin North Am 2015;29:13-28. 